\title{
A test sheet generating algorithm based on intelligent genetic algorithm and hierarchical planning (withdrawal notice)
}

Peipei Gu, Zhendong Niu, Xuting Chen, Wei Chen

Peipei Gu, Zhendong Niu, Xuting Chen, Wei Chen, "A test sheet generating algorithm based on intelligent genetic algorithm and hierarchical planning (withdrawal notice)," Proc. SPIE 8784, Fifth International Conference on Machine Vision (ICMV 2012): Algorithms, Pattern Recognition, and Basic Technologies, 87842Q (13 March 2013); doi: 10.1117/12.2021235

SPIE Event: Fifth International Conference on Machine Vision (ICMV 12), 2012, Wuhan, China 


\section{A test sheet generating algorithm based on intelligent genetic algorithm and hierarchical planning (withdrawal notice)}

Proc. SPIE 8784, 87842Q (2013); http://dx.doi.org/10.1117/12.2021235

Online Publication Date: 13 March 2013

Date withdrawn: 22 October 2013

Conference Date: 20-21 October 2012

Conference Location: Wuhan, China

Conference Title: Fifth International Conference on Machine Vision (ICMV 2012): Algorithms, Pattern Recognition, and Basic Technologies

Conference Chairs: Yulin Wang, Liansheng Tan, Jianhong Zhou

P. Gu, Z. Niu, X. Chen, and W. Chen

Beijing Institute of Technology (China)

This paper was presented at the conference indicated above but was previously presented and published in an earlier SPIE proceedings volume. The paper has been withdrawn from Proc. SPIE 8784 by the publisher. The citation for the earlier paper is: Peipei Gu, Zhendong Niu, Xuting Chen, and Wei Chen, "A test sheet generating algorithm based on intelligent genetic algorithm and hierarchical planning," Proc. SPIE 8334, Fourth International Conference on Digital Image Processing (ICDIP 2012), 83343S (May 1, 2012); DOI: http://dx.doi.org/10.1117/12.966822. 